\title{
La révolution mondiale de la consommation alimentaire : I'émergence d'une nouvelle classe moyenne chinoise
}

\author{
Dominique DESJEUX \\ Université Paris Descartes, \\ 12 rue de l'Ecole de Médecine, \\ 75270 Paris Cedex 06 \\ $<$ d.desjeux@argonautes.fr $>$
}

Article reçu le 19 juillet 2012

Accepté le 27 juillet 2012

\begin{abstract}
Revolution in global food consumption: a new emerging middle-class in China

Re-analysing the 1945-1975 period, these three post-war decades of massive economic and strong demographic growths, is a way to understand a large part of the actual evolution encountered in China. Just as Europe did more than a century ago, China woke up and is about to regain the place in the economy it used to occupy at the beginning of the $19^{\text {th }}$ century. In 2009, China was one of the fourth biggest buyers of agricultural lands in the world with Saudi Arabia, the United Arab Emirates and South Korea. Just as Europe did in 1945, over the last three decades China has built coastal infrastructures such as harbours, highways, airports, encouraging the mobility of people, goods and information. Cities and buildings have developed; there has been industrialization, supply of goods and services to consumers... and finally development of a middle class, especially on the East coast. Or more precisely two middle classes - an upper middle class (105 millions of consumers, with an income of $4800 \$$ to $12500 \$ /$ year) and a lower middle class (190 millions of consumers, with an income of $3000 \$$ to $4800 \$ / y e a r)-$, both responsible for the actual evolution of food consumption in China. Traditional consumption of rice is decreasing, just as potatoes did after the 60's in France; meat and milk consumption (pork, chicken) are soaring. Consequently, the explosion of demand and changes in diets are increasing the strains on world agricultural commodity markets. Nobody knows if China is living the last days of these Golden Years (its gross domestic product (GDP) drop from $14 \%$ to $8 \%$ between 2007 and 2012) or if it is just a step before a new take-off based this time on Central China, after the one based on the East coast. And what about the consequences of the ageing of the Chinese population and of the "one child" policy on future changing lifestyles?
\end{abstract}

Key words: China, middle class, consumption, evolution, rice, meat

taires en dollars est à 100. Quatre ans plus tard, il est déjà monté à 140 . Entre 1998 et 2003, la consommation de soja chinoise est passée de $15 \%$ à $20 \%$ de la consommation mondiale.

\section{Le modèle chinois des "Trente glorieuses": la mise en place des conditions logistiques de la révolution consumériste}

Les Trente glorieuses correspondent à une phase de reconstruction, de « build- ing cycle " pour reprendre l'expression d'Elie Cohen dans L'État Brancardier publié en 1989 pour décrire la période de croissance française entre 1945 et 1975. C'est ce que I'américain Rostow décrit aussi en 1960 sous le terme de "take-off", de décollage. Cette période comprise entre 1945-1975 marque le début de la grande consommation, de l'évolution de la consommation alimentaire au profit des protéines notamment, et donc de la demande en production animale, au détriment des féculents ou des légumes. Plus généralement, c'est celle du changement de mode de vie des Européens de I'Ouest associé à la montée d'une classe

Pour citer cet article : Desjeux D. La révolution mondiale de la consommation alimentaire : l'émergence d'une nouvelle classe moyenne chinoise. OCL 2012 ; 19(5) : 299-303. doi : 10.1684/ocl.2012.0472 
moyenne de consommateurs comme en Chine à peine dix ans plus tard en 1980. L'hypothèse est simple : réanalyser ce que nous avons connu dans les années 1960 peut nous permettre de comprendre une part non négligeable de l'évolution de la Chine contemporaine, au-delà des différences idéologiques et des différences de systèmes politiques, et par là peut nous aider à anticiper les évolutions du marché mais aussi les incertitudes qui pèsent sur cette évolution au regard de I'histoire depuis 200 à 300 ans.

En effet, si on remonte encore plus en amont dans I'histoire, on découvre que cette période de take-off renvoie au début du $\mathrm{XIX}^{\mathrm{e}}$ siècle pendant lequel I'Angleterre grâce à son charbon, au textile américain et à la machine à vapeur associée aux métiers à tisser a réussi le premier décollage industriel moderne. Décoller en premier lui a donné une position hégémonique pendant un siècle, ce qui ne veut néanmoins pas dire sans compétiteurs (et notamment la France, les États-Unis, leur toute récente ancienne colonie, et l'Allemagne).

Cette " pax » britannica n'a pas supprimé les guerres et tout particulièrement celles de conquête ou les conflits militaires, dont la Guerre de I'Opium suivie des Traités inégaux avec la Chine vers 1850 . Ces traités ont par la suite, et pour longtemps, fortement diminué ses capacités de croissance économique. L'occupation de la Chine par le Japon entre 1937 et 1945 n'a fait que renforcer cette tendance. Les séquelles commerciales, politiques et nationalistes entre le Japon et la Chine s'en font encore sentir aujourd'hui : le conflit sur les terres rares nécessaires à la fabrication des nouvelles technologies vient de nous le rappeler. Cela n'empêche pas le Japon d'être l'un des premiers partenaires économiques de la Chine. Pour les plus jeunes générations occidentales, tout ceci paraît être de I'histoire ancienne. Un des objectifs de cet article est de rappeler le retour permanent de I'histoire et de ses contentieux dans les négociations commerciales internationales et dans les tentatives de prospective.

La " pax britannica " a cependant limité les guerres de grandes ampleurs entre 1815, fin des guerres napoléoniennes, et 1913, veille de la première guerre mondiale. Le risque de guerre réapparaît aujourd'hui du fait de l'augmentation de la compétition internationale pour I'accès aux terres agricoles, aux matières premières, à l'énergie et aux fonds sousmarins pour les nodules polymétalliques et de la remise en cause du territoire de la "pax americana".

La Chine se retrouve dans une position qui paraît analogue à celle de l'Allemagne entre 1885 et 1914 face au contrôle que I'Angleterre et la France exerçaient, grâce à leurs colonies, sur l'accès à toutes les matières premières nécessaires au développement économique du "centre ». En 2012 la Chine est en train de construire trois porte-avions pour contrôler la circulation dans la mer de Chine et revendique au sud des îles riches en fonds marins polymétalliques mais qui sont aussi disputées par les Philippines ou le Vietnam. En mai 2012, la Chine a envoyé un navire de guerre devant les îles Huangyan (en Chinois) et Scarborough shoal (en Anglais) pour bien indiquer que cette île lui appartenait. Tout cela reviendrait à faire revivre la " pax sinica " de I'empire Qing sur I'Asie du sud-est entre le $X V I I^{\mathrm{e}}$ siècle et le début du $X V I I{ }^{e}$ siècle, ce qui, à court terme, revient à reprendre de fait une partie de la zone d'influence des Américains. Ceci ne signifie pas qu'il y aura la guerre mais que cela rentre dans les incertitudes qui pèsent sur le développement des marchés et de la circulation maritime des marchandises. Tout cela va dépendre des marges de manœuvre que la Chine va posséder pour développer une "pax sinica » dans ce qu'elle considère comme sa zone d'influence entre mer de Chine du Sud et du Nord, Océan Pacifique et Océan Indien face aux États-Unis, au Japon et à I'Inde.

Un économiste comme Philippe Norel rappelle dans son livre sur L'histoire économique globale publié en 2009 que I'Asie, bien au-delà de la Chine et y compris I'Inde et le Moyen-Orient, représentait probablement $80 \%$ de la production mondiale entre 1750 et 1800. La Chine aurait représenté $25 \%$ de l'économie mondiale. L'historien américain Kenneth Pomeranz, dans $L a$ grande divergence (2010), estime que les économies ouest-européennes et celles chinoises du delta du Yangzi autour de Shanghai étaient équivalentes à la fin du xvIII ${ }^{\mathrm{e}}$ siècle.

C'est I'usage, rappelons-le, d'une nouvelle énergie, le charbon, et de ressources extérieures, le coton, qui a permis le décollage de l'Europe de l'Ouest. Toute ressemblance avec la situation actuelle n'est pas du tout fortuite. La maîtrise non polluante de l'énergie, des matières premières, de l'alimentation, de l'eau et de leur reproduction reste centrale dans tout processus de croissance et de rapport de force encore aujourd'hui entre "États continents ", comme la Chine, les États-Unis ou l'Europe.

On peut malgré tout se demander pourquoi partir de si loin pour répondre à une question plus étroite et très actuelle : en quoi la Chine d'aujourd'hui influence-t-elle la consommation mondiale en général, et en particulier, le marché des oléagineux, et notamment celui du soja ? Parce qu'une partie de la réponse est dans I'histoire. Napoléon aurait dit en 1816 "quand la Chine s'éveillera, le monde tremblera", phrase qui a été reprise par Alain Peyrefitte pour le titre de son livre sur la Chine publié en 1973.

Or la Chine s'est réveillée. Elle est en train de reprendre la place et le poids économique qu'elle avait au début du $X I X{ }^{e}$ siècle avec tout ce que cela implique en termes de tendance longue dans l'évolution des marchés, ce à quoi s'ajoute la compétition pour l'accès aux terres arables dans le monde qui est déjà en train de se durcir. En 2009, les quatre plus gros pays à l'origine des acquisitions de terres agricoles dans le monde étaient I'Arabie saoudite, les Emirats arabes, la Corée du Sud et la Chine pour un peu plus d'un quart des acquisitions comme le montre Jean Yves Carfantan dans Le Monde du 15 avril 2009. On assiste aussi depuis les années 2000 au retour des crises alimentaires liées au manque de riz ou de blé, crises qui avaient fortement diminué, sans disparaître, après les grandes famines des années 1970 en Afrique. C'est en partie la remise à jour de "l'arme alimentaire " dans les négociations internationales. Cette tension est en partie liée à l'augmentation de la demande chinoise, et notamment celle de soja dont elle est le premier consommateur mondial. Elle est liée aussi à l'étroitesse des marchés comme le rappelle Philippe Chalmain dans un livre à la portée de tous, Desépices à l'or noir. L'extraordinaire épopée des matières premières, publié en 2008. II est l'éditeur depuis 1987 de Cyclope, le livre de référence sur les marchés mondiaux dont l'édition de 2011 est sous-titrée : «le printemps des peuples et la malédiction des matieres 
premières ". On ne peut mieux résumer la situation.

Le réveil de la croissance chinoise a suivi un cheminement historique qui rappelle pour une part celui de I'Angleterre au XIX siècle mais surtout celui de I'Europe de l'Ouest après 1945 : mise en place des infrastructures, des routes, des ports, des aéroports et des trains, infrastructures qui créent les conditions du développement de la mobilité des hommes, des biens et de l'information. À partir de cela se développent les villes et les logements, I'industrialisation et une nouvelle offre de biens, la grande distribution, la voiture, une classe moyenne, le tout entraînant une transformation des modes de vie et de consommation. Cette révolution des modes de vie en Chine exerce une influence directe sur l'évolution des marchés de biens alimentaires internationaux. Ce changement représente à la fois un risque et une opportunité systémiques en fonction de la place que chacun occupe comme producteurs, transformateurs, distributeurs et consommateurs, français ou européens.

La mise en place des conditions de la mobilité va permettre de faire venir la main d'œuvre paysanne du centre de la Chine vers les industries de la côte, comme à Shenzhen au Sud, entre 1980 et 2008, année de la crise économique et de la progression de l'augmentation des salaires qui débute vers 2005 et des premières grandes grèves industrielles de l'après-révolution culturelle. Par ailleurs, il faut se souvenir qu'il n'y pas loin de 100000 mouvements sociaux par an en Chine, contre 47000 en 2004 d'après I'International Herald Tribune du 20 mai 2005, ce qui représente une autre forme d'incertitude quant à l'avenir social et politique. On retrouve l'ambivalence du progrès que nous avons connu dans les années 1960 pendant lesquelles le pouvoir d'achat des Français augmentait globalement, en même temps que naissait une nouvelle classe ouvrière issue des campagnes ou de l'immigration.

L'amélioration de la logistique permet aussi la mobilité des matières premières et de l'énergie locale ou importée nécessaire au développement industriel. La Chine bénéficie de la révolution des conteneurs lancée dans les années 1960 aux États-Unis comme le raconte Marc Levinson dans The Box, comment le conteneur a changé le monde, en 2011.
En effet, en 2003, au cœur du take-off chinois, sur les "quatre plus grands terminaux à conteneurs du monde " trois étaient des ports chinois : Hongkong, Shanghai et Shenzhen. En 2005, quatre aéroports entre Canton, Macao, Shenzhen et Hongkong, tous dans un mouchoir de poche au sud de la Chine, se partagent 6 millions de tonnes de fret par an d'après Libération du 10 mai 2005. Infrastructures et conteneurs sont les deux conditions logistiques du commerce mondial, et par là des oléagineux.

Le décollage de la croissance industrielle chinoise s'est fait à l'aide d'investissements extérieurs dont les fonds venaient soit de la diaspora chinoise, soit des banques d'État, soit de financements étrangers. Ceux-ci étaient très contrôlés grâce à la pratique des joint-ventures. La Chine a eu l'intelligence, grâce à un rapport de force qui lui était favorable du fait de l'immense marché qu'elle représentait, de rester majoritaire dans le capital des sociétés. Cependant, investir en Chine n'allait pas sans problème comme le rappelle Tim Clissol dans son livre témoignage Mr China: comment perdre 450 millions de dollars à Pékin après avoir fait fortune à Wall Street, publié en 2006 et dans lequel il raconte comment une partie des fonds investis était détournée en faveur $d^{\prime}$ autres projets que ceux qui étaient prévus par les partenaires chinois. Investir en Chine relève aussi d'une forte incertitude même si le droit et l'organisation de zones industrielles a évolué depuis 10 ans comme j'ai pu l'observer en 2008 à Hangzhou, au sud de Shanghai, dans une enquête sur les TPE, PMI et PME françaises.

Une grande partie des entreprises chinoises sont orientées vers l'exportation et notamment dans le textile, comme en Angleterre au XIX ${ }^{\mathrm{e}}$ siècle. Le développement industriel conduit à son tour au développement des villes et donc de la construction, c'est-à-dire du bâtiment et " quand le bâtiment va tout va " pour reprendre une phrase célèbre des années 1960. "Tout va » du fait des importants effets d'entraînements économiques liés au développement des logements que ce soit en termes de construction, en termes d'équipement des logements avec les installations électriques nécessaires à l'usage de l'électroménager, marché qui a été très bien compris par les sociétés françaises
Legrand et Leroy Merlin pour le bricolage, et de tout ce qui tourne autour de la télévision, des ordinateurs, des jeux vidéo, du téléphone et plus généralement $d^{\prime}$ Internet et des chargeurs de batteries divers que l'on va trouver dans le living ou dans la chambre. Cela concerne aussi tout ce qui va servir à équiper la cuisine, du réfrigérateur au rice cooker électrique pour le riz en passant par le stérilisateur pour la vaisselle. La salle de bain est moins concernée.

Ces biens d'équipement du logement sont proposés grâce à la mise en place de la grande distribution et notamment de Carrefour pour les Français ou de Wal Mart pour les Américains, comme dans la France des années 1960 avec Leclerc, Auchan, Cora ou Carrefour. La mobilité vers ces lieux $d^{\prime}$ acquisition est rendue possible par le développement du métro et des voitures.

En trente ans, on a donc assisté en Chine à la mise en place des infrastructures de base sur la côte Est, d'une industrialisation orientée pour une grande part vers l'exportation mais aussi vers la consommation intérieure avec le textile, les biens de grande consommation, l'électroménager, les NTIC et les voitures, d'une urbanisation associée à une forte migration rurale vers la côte Est, mouvement qui est en train de se retourner, semble-t-il, aujourd'hui en faveur du centre de la Chine, et enfin et surtout à la naissance d'une classe moyenne de consommateurs qui va expliquer, entre autres, la nouvelle demande alimentaire. C'est l'explosion des demandes en protéines animales, laitières et piscicoles et des aliments qui leur sont associés dont le soja.

\section{La nouvelle classe moyenne chinoise : la mutation alimentaire}

D'après Jean Yves Carfantan citant McKinsey dans son livre Le Choc alimentaire mondial : ce qui nous attend demain publié en 2009, il y aurait en Chine une classe moyenne supérieure de 105 millions de consommateurs avec un revenu de $4800 \$$ à $12500 \$$ par an et une classe moyenne inférieure de 190 millions de nouveaux consommateurs avec un revenu de $3000 \$$ à $4800 \$$ soit près de 300 millions de consommateurs. $C^{\prime}$ est cette classe moyenne urbaine 
qui a fait évoluer son mode de consommation alimentaire.

$C^{\prime}$ est ce que j'ai pu observer dans toutes les enquêtes qualitatives sur la vie quotidienne que je mène depuis 15 ans en Chine, de Guangzhou au Sud à Harbin au Nord en passant par Beijing, Shanghai et Hangzhou. Cette évolution est caractéristique des comportements des classes moyennes montantes et jeunes dans le monde. En Chine, cela se traduit par une baisse des consommations traditionnelles comme le riz - les importations de riz ont légèrement baissé en cinq ans même si la Chine reste encore le premier producteur mondial de riz -, le blé et les tubercules. Cela fait écho à la consommation de pomme de terre en France qui s'achetait encore dans les années 1960 par sac de $50 \mathrm{~kg}$.

Par contre la consommation de viande de porc et de poulet explose. Les Chinois consomment en 201037 kg par an de viande de porc par personne contre $27 \mathrm{~kg}$ aux États-Unis et $42 \mathrm{~kg}$ en Europe. Ce qui veut dire, d'après Cyclope 2011, que la Chine est passée de $100000 \mathrm{t}$ d'exportations de viande de porc en 2005 à 350000 t en 2010 . De même, la Chine est passée de $230000 \mathrm{t}$ de volailles importées en 2005 à 350000 t en 2010. Un autre signe par rapport au poulet est le fait que KFC (Kentucky Fried Chicken) possède, en 2010, 3000 points de vente en Chine.

De même, dans un Carrefour de Guangzhou (Canton) que j'ai pu observer, le lait est passé d'une vente confidentielle qui se limitait à un mètre de linéaire à une vente sur plusieurs palettes qui occupe tout un rayon, en 4 ans, entre 2004 et $2008^{1}$.

On retrouve les mêmes évolutions : 2005, 74000 t de lait en poudre entier importé contre 400000 t en 2010 ou encore, 2005, 62000 t de lait écrémé importé contre 100000 t en 2010, soit en 2010 un total de 500000 t de lait importé pour les industries laitières chinoises. Ce chiffre est d'autant plus important que ces industries ont subi une forte baisse de confiance avec les affaires du lait contaminé de 2008 et de 2010. Cette confiance peut revenir un

\footnotetext{
${ }^{1}$ Cf. le film de Dominique Desjeux sur un Carrefour : www.youtube.com/watch?v=ELo GPfOhd3E\&I $r=1$ \&feature $=$ mhee
}

jour mais l'inquiétude des mamans des classes moyennes sur la qualité du lait qui peut menacer leur enfant unique est encore grande et bénéficie aux produits d'importation pour le moment.

Un autre rayon en pleine expansion est celui des alcools et du vin, que les vins soient australiens ou chiliens. Mais les vins de Bordeaux restent très cotés. Le Château Lafitte peut se vendre entre 1500 à $3000 €$ la bouteille. Pour le moment, même si la Chine ne rentre pas encore dans les 10 premiers importateurs de vin, les importations sont en train de monter grâce au marché aux vins de Hongkong qui bénéficie d'une politique douanière favorable, toujours d'après Cyclope 2011.

La montée des produits protéinés, viande et lait génère une explosion de la demande en soja, le tourteau de soja étant une des nourritures de base des animaux. Ce qui est clair d'après Cyclope 2011 c'est que la production de soja en Chine (par ailleurs une culture traditionnelle, ceci pouvant expliquer cela) stagne. J'ai plusieurs fois observé, bien que ce ne soit pas mécanique, qu'il était plus facile $d^{\prime}$ introduire une culture nouvelle que de moderniser une culture traditionnelle : le riz des Hauts Plateaux malgaches dans les années 1970 ou le manioc dans le Pool congolais en sont autant d'illustrations. En revanche, les importations de graines de soja sont passées de 2005 à 2010 de $28 \%$ à $55 \%$ des importations mondiales. La Chine est devenue pendant la même période, en 5 ans, le premier producteur de tourteaux au monde avec 43 millions de tonnes de tourteaux contre 36 millions pour les États-Unis. Les importations sont estimées à 50 millions pour 2011.

Les cours du marché du soja dépendent donc de la montée de la classe moyenne chinoise qui consomme de plus en plus de produits carnés, ce qui pousse à l'importation de graines qui à leur tour sont transformées en tourteaux de soja. Cette tendance de la consommation conduit plutôt pour le moment à une hausse des cours et ceci d'autant plus qu'une partie du soja, 5 à $10 \%$ dans le monde, sert d'agro-carburant et donc réduit la taille du marché consacré à I'alimentation animale. De plus, le soja est toujours dépendant des incertitudes des bonnes ou mauvaises années agricoles sans oublier les incertitudes politiques liées aux embargos comme celui de la Chine en 2010 sur les huiles de soja d'Argentine.

Un autre produit controversé pour des raisons écologiques et diététiques fait de la Chine le premier importateur mondial, I'huile de palme. II est probablement l'indicateur d'une double évolution des modes de vie qui concernent les jeunes couples des classes moyennes supérieures qui travaillent et ont un enfant, voire pas d'enfant, comme les DINKS (Double Income No Kids) qui émergent dans les grandes villes américaines dans les années 1980, et que I'on va trouver en Chine à Beijing ou Shanghai, qu'ils soient homo- ou hétérosexuels. Ils ont moins le temps de faire la cuisine et donc vont davantage au restaurant et surtout achètent plus de plats tout préparés riches en huile. Un autre grand usage est celui des cosmétiques qui est un marché en pleine expansion en Chine, même si la diffusion du maquillage ne va pas de soi, le maquillage des jeunes pouvant être considéré comme négatif ou immoral par les générations plus âgées.

À ces produits végétaux, on pourrait ajouter le poisson produit par aquaculture. En 1986, la Chine importait moins de $300000 \mathrm{t}$ de poisson d'aquaculture contre près de 4 millions de tonnes en 2008. Ils sont nourris avec de I'huile de poisson. On retrouve l'effet "Trente glorieuses " et la montée d'une classe moyenne qui consomme plus de produits protéinés.

Au final, la Chine joue un rôle majeur sur quatre marchés d'importation, celui de la viande de porc, celui du poulet, celui du lait et celui associé du soja en graine. Mais la Chine joue aussi un rôle dans $d^{\prime}$ 'autres productions qui correspondent aux demandes de sa classe moyenne montante, comme le sucre ou le vin. À l'inverse, le café reste un produit d'exception, réservé aux consommateurs branchés. II faudra attendre pour voir si on assiste un jour à un effet Starbuck sur la consommation de café comme on a peut-être eu un effet KFC sur la consommation de poulet. Le cacao semble inexistant. Le thé reste un produit local et d'exportation mais semble concurrencé dans la consommation des plus jeunes par divers sodas.

II reste cependant quatre grandes incertitudes: I'évolution des mouvements sociaux à la campagne et en ville, les effets et les conséquences de la 
pollution sur la qualité de vie des chinois, le risque ou non d'une bulle immobilière ou d'un crash boursier, et la durée de la mise en place d'un système de protection sociale publique ou privée qui en limitant les risques de prise en charge sanitaire et en garantissant la retraite permettrait de limiter l'épargne de précaution en vue de la réorienter vers le crédit et la consommation intérieure. Cependant, à la vue des effets pervers du crédit avec la crise des subprimes de 2007-2008, puis la crise des dettes souveraines en Europe, on peut se demander si cela représente bien la seule alternative à mettre en place.

\section{De la Chine à la France}

À moyen terme et de façon simplifiée, on observe donc le modèle systémique suivant : la montée des classes moyennes supérieures en Chine entraîne la montée, irrégulière ou volatile, des cours des matières premières agricoles et non agricoles. L'augmentation du coût des intrants, notamment le pétrole et le soja pour I'alimentation du bétail, entraîne une inflation des prix des produits alimentaires qui à leur tour touchent les classes sociales les plus défavorisées, celles pour qui la part de la consommation alimentaire dans le budget familial est la plus importante en France, mais aussi en Chine.

Or, les classes moyennes françaises ont un pouvoir d'achat limité du fait de la montée des dépenses contraintes comme celles liées au logement, à la mobilité et surtout depuis 10 ans, avec la consommation des nouvelles technologies qui tournent autour de $8 \%$ en moyenne par ménage pour monter à $17 \%$ pour les ménages les plus démunis. Ces dépenses contraintes, ou dépenses "obligatoires ", et la "baisse de pouvoir d'achat ", - non pas au sens technique des économistes de baisse de revenu mais au sens d'une baisse de capacité à faire face à toutes les dépenses contraintes dont les dépenses alimentaires -, qui leur sont associées, rendent l'augmentation des prix alimentaires encore plus problématique pour les ménages démunis.

$C^{\prime}$ est pourquoi on voit se développer en France des pratiques parallèles ou alternatives comme les AMAP (associations pour le maintien d'une agriculture paysanne), le glanage ou les achats entre pairs sur Internet, pratiques qui jusque-là étaient réservées aux plus pauvres mais qui commencent à toucher les classes moyennes inférieures. Ces pratiques de "consommateurs malins" sous contrainte de pouvoir d'achat sont les symptômes de cette baisse de capacité à consommer en suivant les circuits de distribution classiques. Les salaires bas des salariés chinois permettent de compenser une partie de cette perte grâce aux bas prix du textile. Cependant, globalement, I'augmentation des coûts des matières premières agricoles et non agricoles menace les strates sociales le plus fragiles.

\section{Conclusion}

On ne sait pas encore si la Chine est en train de toucher à la fin des "Trente glorieuses ", la croissance du PIB étant passé en 5 ans de $14 \%$ à $8 \%$ en 2012 ou si ce n'est qu'une étape avant un nouveau rebond économique grâce aux régions centrales qui peuvent servir de base pour un nouveau take-off. On ne sait pas non plus les implications du vieillissement de la population sur l'évolution des modes de vie ni celles de I'enfant unique.

Cependant, ce qui paraît probable, c'est que cette tendance à la hausse des cours, même irrégulière, risque de durer quand on sait que la classe moyenne supérieure mondiale qui intègre celles des BRICS (le Brésil, la Russie, I'Inde, la Chine et l'Afrique du Sud) et des pays développés, représentait 200 millions de personnes en 2000 et 560 millions en 2009. La Chine en représente $20 \%$ et elle continue à progresser. La Chine en particulier, et I'Asie du Sud-Est en général, jouent donc un rôle central sur les marchés des matières premières agricoles. Observer l'évolution des modes de vie de ces classes moyennes constitue une des entrées stratégiques pour comprendre l'évolution de ces marchés, au-delà des incertitudes climatiques, politiques, écologiques, militaires ou de rapports de forces au sein de chaque filière.

Conflits d'intérêts : aucun 